\title{
Quantitative Soft X-ray Microscopy of Soft Matter
}

\author{
H. Ade
}

Department of Physics, North Carolina State University, Raleigh, NC 27695-8202

Near Edge X-ray Absorption Spectroscopy (NEXAFS) in a Scanning Transmission X-ray Microscopes (STXM) provides quantitative compositional information with a spatial resolution of about $35 \mathrm{~nm}$ and minor radiation damage [1-4]. This combination of capabilities is unique, overcoming the disadvantage of having to use a synchrotron radiation facility to provide a tunable source of X-rays. Since 1992, NEXAFS has been used for a wide range of soft materials and applications range from the characterization of polymer blends, microballoons, protein adsorption, polyurethanes, to the in-situ characterization of microgels, environmental samples and electrochemical processes [5]. The presentation associated with this abstract will provide an overview of the field and outline future opportunities. Here, recent applications of NEXAFS microscopy to organic electronic devices [6-12] and to microgels $[13,14]$ are used to exemplify the capabilities.

Organic devices have the potential to significantly contribute to solving the world's energy needs, both by providing conversion of solar energy to electricity in solar cells (SC) and in achieving improved efficiency through the use of light emitting diodes. There is, however, a great need to correlate compositional inhomogeneities, intrinsically necessary in bulk-heterojunction organic devices, to SC device performance. Furthermore, the purity of the domains play a major role in how the devices actually work, yet very little is know about that. An example is poly(3-hexylthiophene) (P3HT) and (6,6)-phenyl $\mathrm{C}_{61}$ butyric acid methyl ester (PCBM) blends, in which the electron donor material (P3HT) and the electron acceptor material (PCBM) are blended together in order to optimize both exciton separation and charge transport. Recent NEXAFS microscopy investigations have shown that the P3HT domains in a P3HT:PCBM blend are not pure, even though that is commonly assumed. Compositional characterization of the compositional gradient near a PCBM crystal showed that the residual PCBM concentration near the crystal boundary is 20\% [12].

NEXAFS microscopy has also the capability to investigate fully hydrated or solvated materials such as microgels. Under suitable conditions microgels can swell to many times their original volume and hence act like 'nano-sponges', thus offering potential applications ranging from drug delivery and heavy metal sequestration, to sensors and smart particulate emulsifiers [13,14]. Microgels are employed in their swollen state and in situ characterization under such conditions is desirable for the complete understanding of their properties. NEXAFS microscopy can directly image swollen and unswollen microgel particles in aqueous solutions and characterize their charge state individually in situ [13,14]. Lightly cross-linked sterically-stabilized poly(2-vinylpyridine) latexes microgel particles were imaged in both their non-swollen and swollen states by adjusting the solution $\mathrm{pH}$. In addition, the effects of salt concentration was assessed. Spectroscopic analysis of the $\mathrm{N} 1 \mathrm{~s} \rightarrow \pi^{*} \mathrm{~N}=\mathrm{C}$ transition in the P2VP showed a characteristic spectral shift when the P2VP was protonated and demonstrated that the microgel particles were fully protonated at $\mathrm{pH}=3$, irrespective of the salt concentration. These results directly elucidate the underlying mechanism for deswelling at high salt concentration (see Fig 2), as the reduced size could now be ascribed to screening of the protonated polymer by the salt. 
References

[1] Ade, H., Zhang, X., Cameron, S. et al., Science 258 (1992) 972.

[2] Kilcoyne, A. L. D., Tyliszczak, T., Steele, W. F. et al., J Synchrotron Radiat 10 (2003) 125.

[3] Ade, H. and Hitchcock, A. P., Polymer 49 (2008) 643.

[4] Ade, H. and Stoll, H., Nat Mater 8 (2009) 281.

[5] Ade, H. and Hitchcock, A. P., Polymer 49 (2007) 643.

[6] McNeill, C. R., Watts, B., Thomsen, L. et al., Nano Lett 6 (2006) 1202.

[7] McNeill, C. R., Watts, B., Thomsen, L. et al., Small 2 (2006) 1432.

[8] McNeill, C. R., Watts, B., Thomsen, L. et al., Macromol. 40 (2007) 3263.

[9] McNeill, C. R., Watts, B., Thomsen, L. et al., Macromol. 42 (2009) 3347.

[10] Burke, K. B., Belcher, W. J., Thomsen, L. et al., Macromol. 42 (2009) 3098.

[11] McNeill, C. R., Watts, B., Thomsen, L. et al., Nanotechnology 19 (2008) 424015.

[12] Watts, B., Belcher, W. J., Thomsen, L. et al., Macromol. ASAP (2009)

[13] Fujii, S., Armes, S. P., Araki, T. et al., J. Am. Chem. Soc. 127 (2005) 16808.

[14] Fujii, S., Dupin, D., Araki, T. et al., Langmuir 25 (2009) 2588.
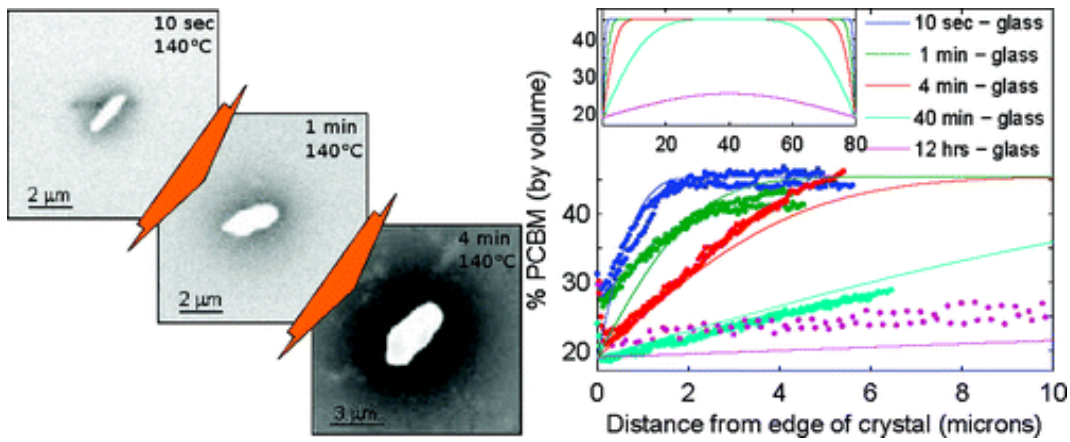

FIG. 1. NEXAFS microscopy has been used to quantitatively map the composition of P3HT:PCBM blend films in the vicinity of PCBM crystals formed during annealing at $140{ }^{\circ} \mathrm{C}$. The observed PCBM concentration profiles around these crystals have been fitted to Fick's second law of diffusion and the diffusion constant found to be $2.5 \times 10^{-14} \mathrm{~m}^{2} \mathrm{~s}^{-1}$. The PCBM concentration at the crystal boundary was found to be $19 \%(\mathrm{v} / \mathrm{v})$ [12].

a

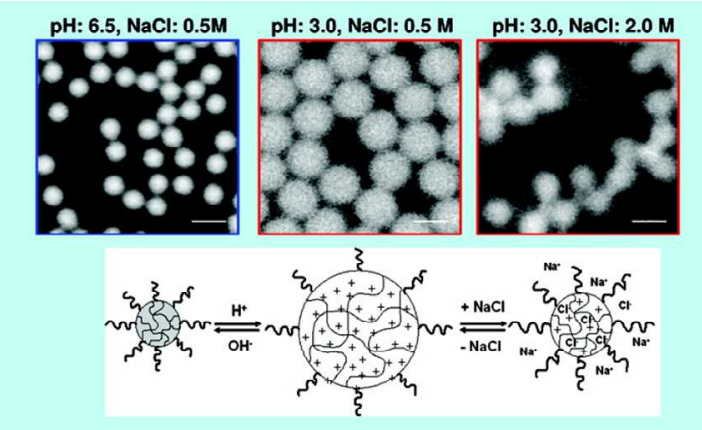

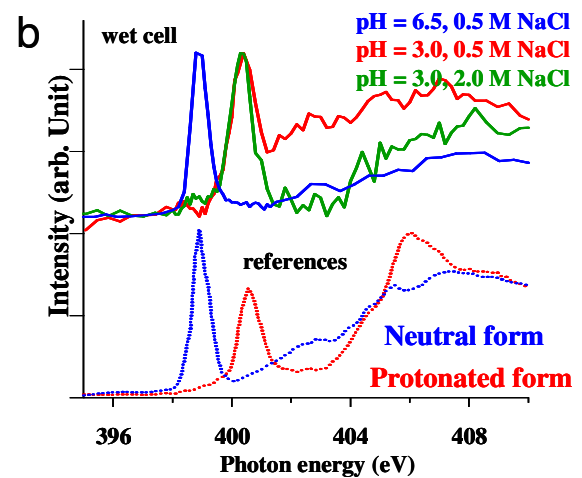

FIG. 2. (a) Optical density images of aqueous dispersions of nanocomposite microgel particles as a function of $\mathrm{pH}$ and salt concentration and a schematic of these cationic microgel particles (b) NEXAFS spectra of an individual microgel particle and reference spectra at $\mathrm{pH} 10$ and ph 2.5, respectively [14]. 